\section{Vitreous levels of placental growth factor correlate with activity of proliferative diabetic retinopathy and are not influenced by bevacizumab treatment}

E Al Kahtani ${ }^{1,4}$, Z Xu², S Al Rashaed ${ }^{1}$, L Wu ${ }^{2}$, A Mahale ${ }^{1}$, J Tian³ ${ }^{3}$ EB Abboud ${ }^{1}$, NG Ghazi ${ }^{1}$, I Kozak ${ }^{1}$, V Gupta ${ }^{1}$, JF Arevalo ${ }^{1,2}$ and EJ Duh ${ }^{2,4}$

\begin{abstract}
Purpose Placental growth factor (PIGF) is a member of the VEGF family that plays an important role in experimental models of diabetic retinopathy and retinal neovascularization. We aimed to investigate whether vitreous levels of PIGF correlated with proliferative diabetic retinopathy (PDR) status, VEGF levels, and bevacizumab treatment. We also analysed PDR membranes to confirm the presence of the PIGF receptor, FLT1, in endothelial cells.

Methods This was a case-control study: undiluted vitreous fluid samples were obtained from 28 active PDR patients without preoperative bevacizumab treatment, 21 active PDR patients with preoperative bevacizumab treatment, 18 inactive PDR patients, and 21 control patients. PIGF and VEGF levels in samples were determined by enzyme-linked immunosorbent assay. Immunohistochemistry for FLT1 was performed on human PDR membranes.

Results Compared to control, vitreous PIGF levels were higher in both active PDR without bevacizumab $(P<0.0001)$ and with bevacizumab $(P<0.0001)$. There was no significant difference in PIGF between active PDR patients without and with bevacizumab $(P=0.56)$. Compared to active PDR, PlGF levels were significantly reduced in inactive PDR $(P=0.004)$. PIGF levels were highly correlated with VEGF levels in active PDR. VEGFR1 was expressed in endothelial cells in human PDR membranes.

Conclusion The strong correlation of PIGF levels with PDR disease status and expression of FLT1 in human PDR membranes suggest
\end{abstract}

that PIGF has a pathogenic role in proliferative diabetic retinopathy. Therapeutic targeting of PIGF with agents like aflibercept may be beneficial.

Eye (2017) 31, 529-536; doi:10.1038/eye.2016.246; published online 25 November 2016

\section{Introduction}

Diabetic retinopathy is a major cause of vision loss globally, and its global impact is expected to increase significantly due to the increasing prevalence of diabetes mellitus and the increasing life span of individuals with diabetes. ${ }^{1}$

In Saudi Arabia, diabetes has become far more prevalent in the past two decades, with a prevalence of $23.7 \%$ in adults, ten-fold higher than in $1980 .^{2}$ This is associated with an overall 19.7-31.3\% prevalence of diabetic retinopathy. ${ }^{3,4}$ Proliferative diabetic retinopathy (PDR) is the most common cause of severe visual loss in diabetes, and has an estimated worldwide prevalence of 17 million individuals. ${ }^{5}$ Neovascularization is the hallmark of PDR and often results in vitreous haemorrhage and tractional retinal detachment, which can lead to severe loss of vision. The identification of vascular endothelial growth factor (VEGF) as a major stimulus for PDR has led to increasing clinical management of this condition with antiVEGF therapies. However, it is highly likely that other important angiogenic growth factors are involved in this condition, ${ }^{6}$ which could serve as additional targets for therapy.

A significant body of evidence in basic research and pre-clinical research has emerged
${ }^{1}$ King Khaled Eye Specialist Hospital, Riyadh, Kingdom of Saudi Arabia

${ }^{2}$ Wilmer Eye Institute, Johns Hopkins University School of Medicine, Baltimore, MD, USA

\section{${ }^{3}$ Department of}

Biostatistics, Johns Hopkins University School of Medicine, Baltimore, MD, USA

\section{Correspondence:}

E Al Kahtani, King Khaled Eye Specialist Hospital, Riyadh, Kingdom of Saudi Arabia

Tel: +966(11) 4821234

Ext 3760;

Fax: $+966(11) 4821234$ Ext 2044

E-mail: ekahtani@kkesh. med.sa or EJ Duh, Wilmer Eye Institute, Johns Hopkins University School of Medicine, $400 \mathrm{~N}$. Broadway, Smith Building, Room 3011, Baltimore, MD 21287, USA

Tel: +1 410614 3388;

Fax: +1 4105025244

E-mail: eduh@jhmi.edu

${ }^{4}$ These authors contributed equally to this work.

Received: 6 December 2015 Accepted in revised form: 30 September 2016 Published online: 25 November 2016 
supporting the pathogenic role of placental growth factor (PlGF). PlGF is a member of the VEGF family, and binds the VEGF receptor FLT1. In contrast to VEGF, PIGF is not required for normal health, including embryonic development. ${ }^{7,8}$ Similar to VEGF, PIGF is upregulated by hypoxia. Studies of PIGF knockout mice indicate that PlGF is not required for physiological angiogenesis, but does play a critical role in angiogenesis under pathological conditons. ${ }^{8}$ Among its cellular activities, PIGF has direct effects on vascular endothelial and mural cells in promoting angiogenesis. ${ }^{7}$ Of particular importance for PDR, PIGF has been found to promote retinal neovascularization in a mouse model of ischaemiainduced retinopathy. ${ }^{9}$ Interestingly, a recent study has also implicated PlGF in earlier diabetic retinopathy, including retinal vascular leakage. ${ }^{10}$

The clear pathogenic role of VEGF in PDR has led to the increasing use of anti-VEGF compounds in this setting, especially when there is accompanying neovascular glaucoma or vitreous haemorrhage. ${ }^{11}$ Currently, three anti-VEGF drugs are in common use, bevacizumab (Avastin; Genentech Inc., San Francisco, CA, USA), ranibizumab (Lucentis; Genentech Inc.), aflibercept (Eylea; Regeneron Pharmaceuticals Inc., Tarrytown, NY, USA). ${ }^{12}$ Although all three effectively bind VEGF and reduce its ocular levels, the three have important differences, including their structure and VEGF-binding affinity. An additional difference between the drugs is their growth factor specificity. Aflibercept in particular is a soluble fusion protein of the extracelluar domain of VEGFR-1 and VEGFR-2 and the Fc region of immunoglobulin G (IgG), allowing it to efficiently bind both VEGF-A and PIGF. ${ }^{12}$ This additional ability to bind PIGF could potentially result in differential effects of aflibercept compared to the more specific anti-VEGF drugs. On the other hand, although bevacizumab and ranibizumab are specific for VEGF, studies suggest that bevacizumab treatment can influence ocular levels of other cytokines, which may be attributable to the acute reduction in VEGF levels. ${ }^{13,14}$ Since VEGF is a known regulator of PlGF expression, ${ }^{15,16}$ it is conceivable that anti-VEGF treatment could modulate PlGF levels.

PlGF has been found to be upregulated in patients with PDR, 17,18 suggesting that it might be involved in promoting this condition. However, further studies are desirable to support and strengthen a pathogenic role for PIGF in PDR. To this end, we collected vitreous samples from patients with and without PDR. Our objective was to evaluate PIGF in three respects-correlation with level of PDR disease activity, active vs quiescent; potential influence of anti-VEGF; correlation with VEGF levels. In addition, we examined the presence and localization of the PIGF receptor FLT1 in PDR membranes.

\section{Materials and methods}

\section{Study participants}

Patients undergoing vitreoretinal surgery at the King Khaled Eyes Specialist Hospital (KKESH) were recruited for the study. A total of 67 eyes of 67 patients with type 2 diabetes who were affected by PDR were enrolled in this study. The patients were divided into four groups. The eyes were classified as active PDR if there was perfused iridic or pre-retinal neovascularization visible on examination at the time of clinical examination and surgery. Based on the presence of the above features, 49 eyes were classified as active PDR, and 18 as inactive PDR. The inactive PDR cases did not receive preoperative bevacizumab. The active PDR cases were classified according to the administration of preoperative bevacizumab, within 7 days of surgery. The control group consisted of 21 eyes of 21 patients with no DR and control ocular conditions who underwent vitreoretinal surgery; the ocular conditions were nonangiogenic disease processes, including macular hole, rhegmatogenous retinal detachment, vitreomacular traction, and dropped nucleus. Sample sizes were chosen based on two previous studies of vitreous PlGF. ${ }^{17,18}$

The study followed the tenets of the Declaration of Helsinki. The Institutional Review Boards at the Johns Hopkins School of Medicine and the King Khaled Eye Specialist Hospital approved the study protocol, and study participants gave informed consent to the treating surgeon.

\section{Sample collection}

For vitreous specimens, undiluted vitreous fluid samples were obtained at the time of vitreoretinal surgery. All patients underwent pars plana vitrectomy with either 23-gauge or 25-gauge vitrectomy technique. Undiluted vitreous samples were first obtained prior to switching the infusion line on, collected into sterile tubes, and transferred immediately to ice. Vitreous specimens were stored at $-80^{\circ} \mathrm{C}$ and kept frozen until analysis. PDR membranes were also collected at the time of vitreoretinal surgery. The excised membranes were fixed in $10 \%$ formalin.

\section{Measurement of vitreous PlGF and VEGF by ELISA}

The levels of PIGF and VEGF in human vitreous were measured using commercial ELISA kits (Duosets from R\&D, Minneapolis, MN, USA). The minimum detectable dose (MDD) was determined with an assay recommended by R\&D. Specifically, we calculated MDD by adding two standard deviations to 
the mean optical density of 20 zero standard replicates, then calculating the corresponding concentration from the standard curve. The MDDs for PlGF and VEGF Duosets were 3.7 and $24.8 \mathrm{pg} / \mathrm{ml}$, respectively. The ELISA procedures were performed according to the instructions from the manufacturer.

\section{Immunohistochemistry}

Deparaffinized sections were boiled in $1 \times$ target retrieval solution (Dako, Capenteria, CA, USA) for $20 \mathrm{~min}$. After blocking in 5\% normal goat serum diluted in PBS, the sections were incubated with anti-FLT1 antibody $(1: 200$, Santa Cruz, Dallas, TX, USA), anti-CD31 antibody (1 : 100, Abcam, Cambridge, MA, USA) or the isotype- and concentration-matched normal IgG control overnight at $4{ }^{\circ} \mathrm{C}$. After three washes with PBST (PBS plus $0.1 \%$ Tween $20)$, sections were then incubated with the secondary biotinylated goat anti-rabbit or anti-mouse IgG (1:2000) for $1 \mathrm{~h}$ at room temperature. Sections were then detected by the alkaline phosphatase detection system (Vectastain ABC-AP kit, Vector Laboratories, Burlingame, CA, USA) and a blue reaction product was produced by incubating sections with alkaline substrate (Vector blue AP substrate kit III, Vector Laboratories). Sections were also stained with haematoxylin and eosin.

\section{Statistical analysis}

Wilcoxon sum rank test was used to compare the median of PIGF and VEGF between groups. Spearman correlation was calculated to study the correlation between the levels of VEGF and PIGF in active PDR without the bevacizumab group. Differences were considered statistically significant at $P<0.05$.

\section{Results}

Increased vitreous levels of PlGF in PDR patients and lack of effect of bevacizumab treatment on the vitreous levels of PlGF in active PDR patients

We harvested undiluted vitreous samples from the eyes of patients with active PDR, inactive PDR and control conditions (no DR). PlGF was undetectable in the control group and assigned the value of $3.7 \mathrm{pg} / \mathrm{ml}$, representing the limit of detection. Vitreous PIGF levels were significantly increased in both the active PDR group without bevacizumab $(24.8 \mathrm{pg} / \mathrm{ml}$, range: 3.7-773.5; $P<0.0001)$ and with bevacizumab $(11.5 \mathrm{pg} / \mathrm{ml}$, range: 3.7-211.4; $P<0.0001$; Figure 1 and Table 1). The vitreous PIGF levels in the inactive PDR group (which did not receive pre-operative bevacizumab) were significantly reduced $(11.0 \mathrm{pg} / \mathrm{ml}$, range: $3.7-96.9 ; P=0.004)$ compared to the active PDR group without bevacizumab (Figure 1 and Table 1). Vitreous PIGF levels in this inactive PDR

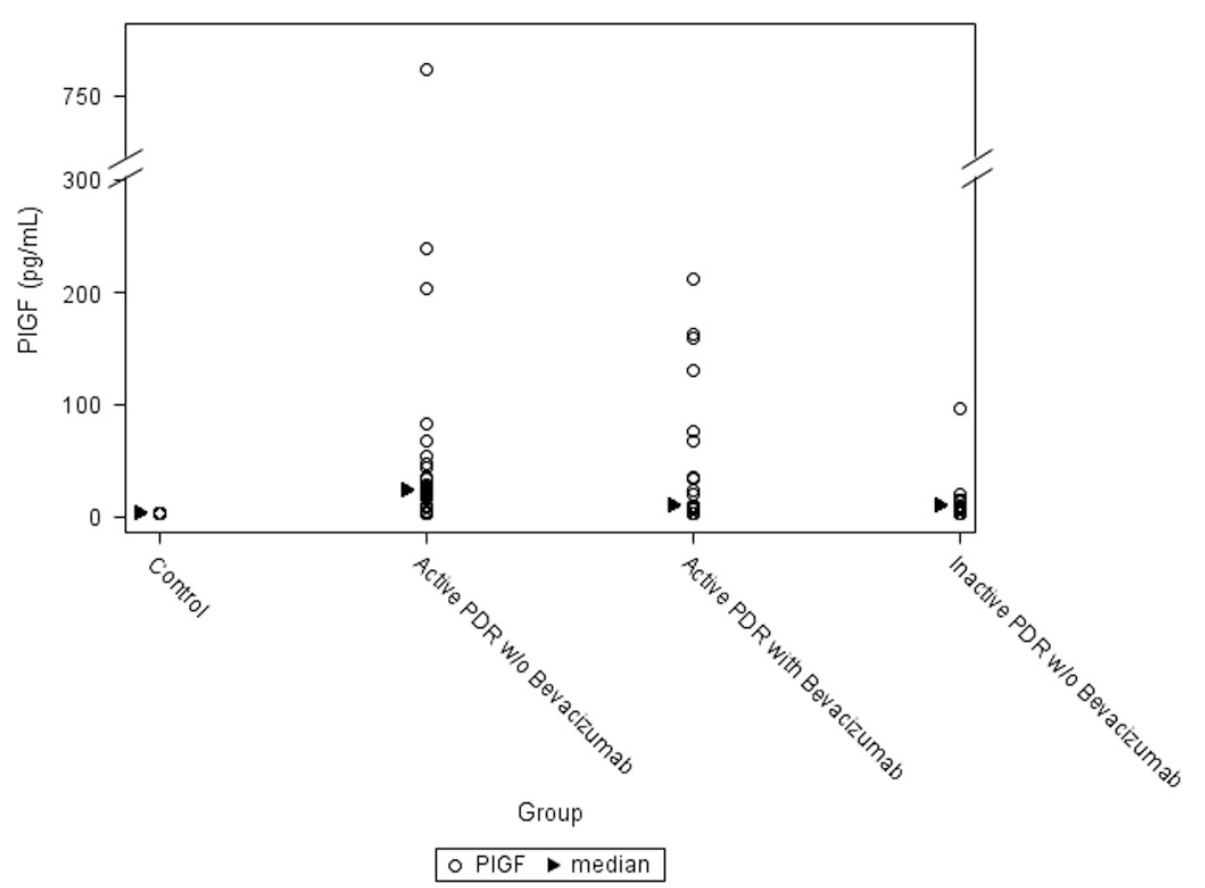

Figure 1 PlGF levels in the vitreous among active PDR without $(n=28)$ or with $(n=21)$ bevacizumab treatment, inactive PDR $(n=18)$ and no DR $(n=21)$. 
group were significantly higher than those in the control group $(P<0.0001$; Figure 1 and Table 1$)$.

\section{Vitreous levels of VEGF in PDR patients and relationship of bevacizumab treatment to VEGF and PIGF levels}

Vitreous VEGF levels were significantly increased in the active PDR group without bevacizumab $(265.9 \mathrm{pg} / \mathrm{ml}$, range: 24.8-5853; $P<0.0001$; Figure 2 and Table 1). The vitreous VEGF levels in the inactive PDR group (which did not receive pre-operative bevacizumab) were significantly reduced $(35.6 \mathrm{pg} / \mathrm{ml}$, range: $24.8-735.5$; $P=0.021)$ compared to the active PDR group without bevacizumab (Figure 2 and Table 1). Vitreous VEGF levels in the active PDR group with bevacizumab were below the limit of detection $(24.8 \mathrm{pg} / \mathrm{ml})$, as expected. In contrast, there was no significant difference in vitreous PlGF levels between active PDR patients with and without bevacizumab treatment $(P=0.56$; Figure 2 and Table 1).

\section{Correlation between PIGF and VEGF in the vitreous of active PDR patients without bevacizumab treatment}

We sought next to determine if there was a correlation in the levels of PIGF and VEGF in the vitreous specimens. We found that there is a highly significant correlation between the levels of PIGF and VEGF in active PDR patients without bevacizumab treatment $(r=0.83$, $P=0.0001$; Figures $3 a$ and $b$ ). In order to facilitate visualization of this correlation between levels of PIGF and VEGF, we constructed a graph that excluded the three highest values (3B).

\section{Immunohistochemical analysis of VEGF receptor 1 in PDR membranes}

Using immunohistochemistry, we investigated the expression and localization of the PlGF receptor, FLT1 (also known as VEGFR1), in active PDR membranes. We were specifically interested in whether FLT1 was expressed in vascular endothelial cells by immunohistochemical staining. In PDR membranes, FLT1 expression was detected in vascular endothelial cells, as indicated by corresponding staining with the endothelial cell marker CD31/PECAM-1 (Figure 4).

\section{Discussion}

The discovery of VEGF as a major regulator of diabetic retinopathy has led to the extensive use of anti-VEGF. These medications are often used in the context of PDR, especially in the presence of vitreous haemorrhage or neovascular glaucoma. ${ }^{11}$ However, these anti-angiogenic 


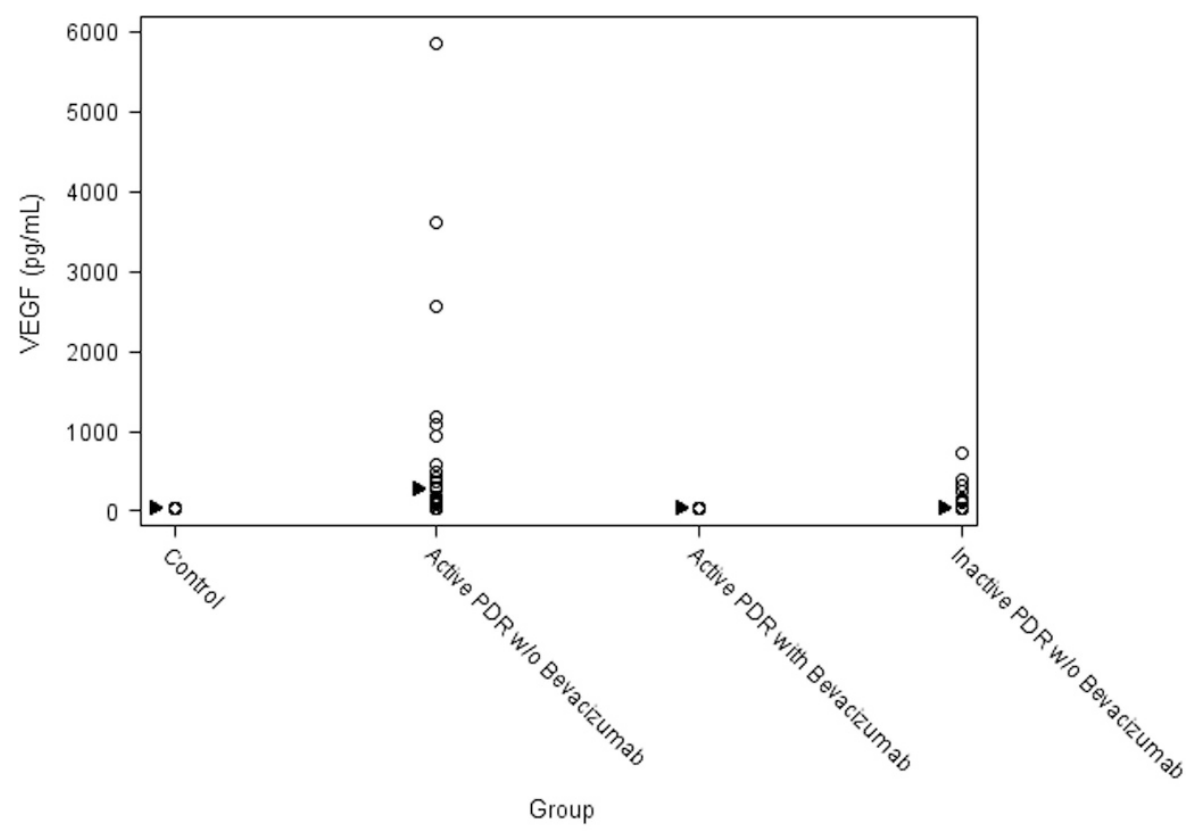

$\circ$ VEGF $>$ median

Figure 2 VEGF levels in the vitreous among active PDR without $(n=25)$ or with $(n=19)$ bevacizumab treatment, inactive PDR $(n=16)$ and no DR $(n=19)$.
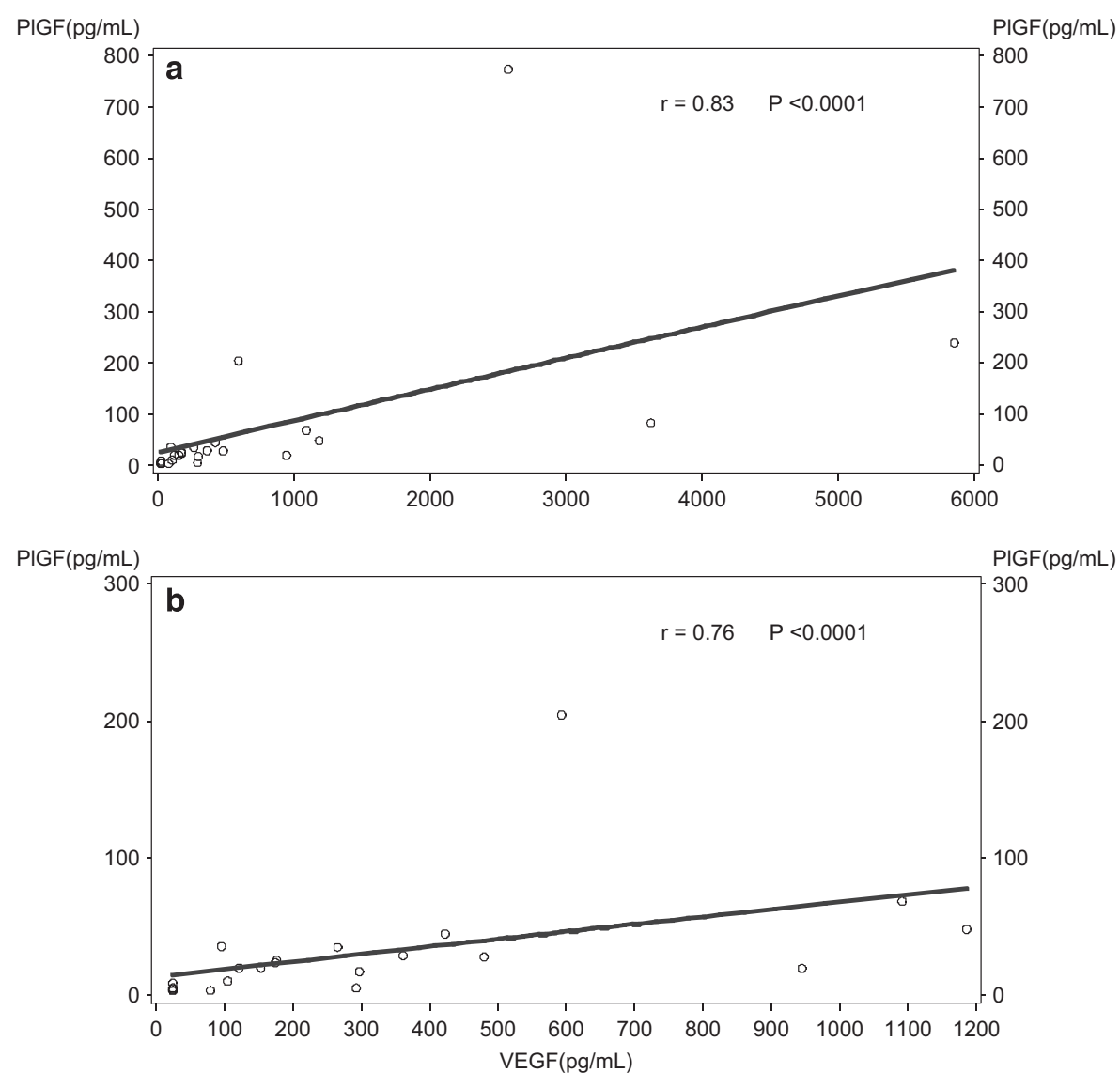

Figure 3 Correlations between vitreous levels of VEGF and PIGF in active PDR without bevacizumab treatment by Spearman's correlation coefficient test, either with inclusion of all values (a) or with exclusion of the three highest values (b). $r$ and $P$ values are shown in the figure. 
therapies still face a number of clinical obstacles and drawbacks, such as short-lived effect and optimal dosing. There are potential safety concerns, in part based on the role of VEGF in maintenance and health of retinal cell types, ${ }^{11}$ and concerns have been raised linking anti-VEGF to retinal atrophy in macular degeneration ${ }^{19}$ and to development of glaucoma. ${ }^{20}$ As a result, it is of great interest to identify additional factors that may play a role in PDR and diabetic retinopathy in general.

Basic research and pre-clinical studies suggest that PIGF could be a major pathogenic molecule in diabetic retinopathy. However, relatively few human studies have been presented to support the role of PlGF in human DR. ${ }^{17,18}$ A very recent study demonstrated that vitreous PIGF levels in patients with neovascular glaucoma associated with ischaemic retinal diseases were even higher than those in PDR patients without NVG. ${ }^{21}$ This correlation of PlGF levels with severity of retinal ischaemia further suggests its pathogenic role. Our current findings that vitreous PIGF levels are reduced in quiescent PDR compared to active PDR further bolsters the likely importance of PlGF in human PDR. In addition, our immunohistochemical studies demonstrating the expression and localization of the PIGF receptor, FLT1, in vascular endothelial cells in human PDR membranes solidify the relevance of elevated PlGF levels for retinal angiogenesis. Strikingly, a previous study found that PlGF intensely localized to the endothelial and perivascular region of newly formed blood vessels of excised fibrovascular preretinal membrane in patients with PDR. However, PIGF was weak or absent in diabetic retina without neovascularization and was absent in normal retina and in diabetic retina following extensive panretinal photocoagulation. ${ }^{17}$

An additional facet of our study was the very strong correlation between vitreous levels of PlGF and VEGF in active PDR. This would be consistent with a common stimulus that upregulates expression of both growth factors. Indeed, studies in mouse models of ischemic retinopathy have implicated HIF- $1 \alpha$ as a transcription factor that induces both VEGF and PIGF in the context of hypoxic retinal disease. ${ }^{22}$

The likely importance of PlGF as an additional pathogenic factor in diabetic retinopathy is interesting in light of the current extensive use of anti-VEGF agents in Saudi Arabia and worldwide and may offer novel opportunities for additional therapeutic strategies. In comparison to anti-VEGF, treatment with anti-PIGF antibody ( $\alpha$-PlGF) might provide therapeutic benefits without the potential side effects of anti-VEGF drugs as noted above. Interestingly, the therapeutic potential of a humanized version of $\alpha$-PIGF (TB403) is being evaluated in cancer patients. ${ }^{23}$ Conceivably, this and future antiPIGF drugs could be considered as new treatment options for both PDR and diabetic macular oedema with improved efficacy.

Recently, aflibercept has been demonstrated to achieve better visual outcomes than bevacizumab and ranibizumab in certain contexts, including a subgroup of patients with diabetic macular edema. ${ }^{24} \mathrm{~A}$ potential differentiating property of aflibercept is its ability to bind PIGF in addition to VEGF, leading to speculation that this characteristic might render aflibercept more efficacious. ${ }^{25,26}$ A recent pre-clinical study has demonstrated the importance of PlGF in experimental diabetic retinopathy. ${ }^{10}$ Particularly relevant to PDR, basic research and pre-clinical studies have demonstrated that PlGF plays a role in promoting the angiogenic phenotype in endothelial cells and in stimulating angiogenesis in vivo, including a mouse model of ischaemia-induced retinal neovascularization. ${ }^{9,27}$

Although aflibercept has a direct effect in binding PlGF, it is plausible that bevacizumab and ranibizumab, via their anti-VEGF effect, might also influence PIGF levels. VEGF has previously been shown to upregulate PIGF expression. ${ }^{15,16}$ Studies indicate that preoperative treatment with bevacizumab may influence vitreous levels of some cytokines and growth factors, including SDF- $1^{13}$ and some inflammatory cytokines. ${ }^{14}$ We were therefore interested in determining whether preoperative treatment with bevacizumab influenced vitreous levels of PlGF. In the current study, we did not find evidence to suggest such an effect.

Although our study demonstrates a strong correlation of PlGF levels with PDR disease status, it does not establish a definitive cause and effect relationship between PIGF and PDR progression, and additional studies will be required. In addition, the findings relate specifically to PDR and not diabetic macular oedema due to the inherent limitations in access to vitreous specimens from the latter condition. With regard to the effect of antiVEGF treatment, it would be helpful to include studies in the future that address the effect of aflibercept, given its known property for binding both PIGF and VEGF.

Finally, we acknowledge the relatively small sample size for some of our groups, although the very low $P$-values in our study strongly support our major conclusions. Larger follow-up studies will likely be useful to solidify these findings.

In conjunction with previous studies, our findings lend further support to the concept that PlGF plays an important role in proliferative diabetic retinopathy. This raises the possibility that aflibercept may provide an added advantage over other current anti-VEGF agents. Further studies will be required to confirm the potential benefit conferred by suppression of PIGF in retinal diseases including proliferative diabetic retinopathy. 

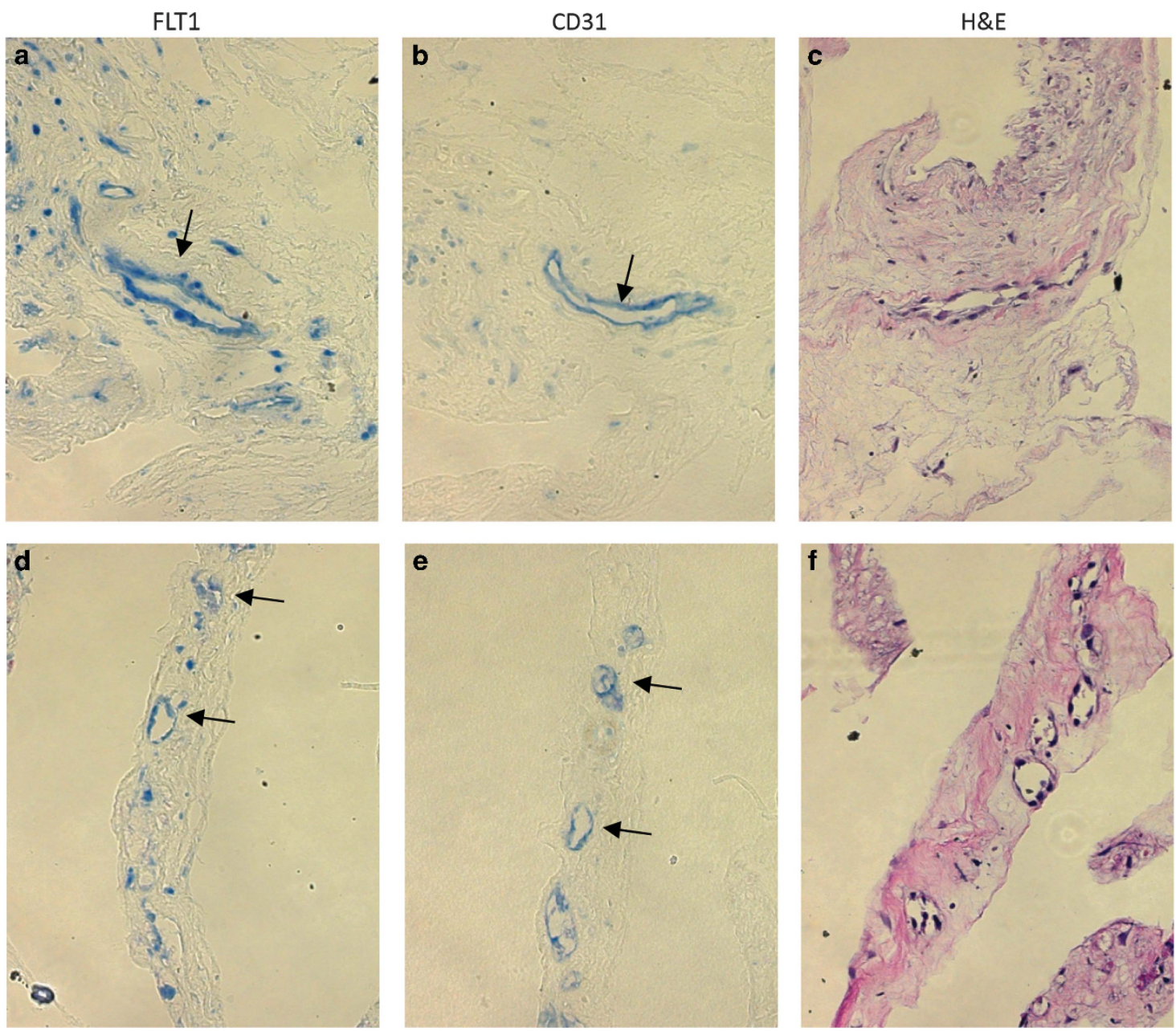

Figure 4 The expression of FLT1 in PDR membranes. (a, b) IHC staining of FLT1 in membranes from active PDR patients. FLT1 is expressed in endothelial cells lining the lumen of blood vessels (black arrows). (c, d) CD31 staining confirmed the distribution of microvascular endothelial cells in adjacent sections from PDR membranes that were used in (a) and (b). (e) H\&E staining in adjacent sections from PDR membranes that were used in (a) and (b). The images were taken using $\times 20$ objective.

\section{Summary}

\section{What was known before}

- Placental growth factor (PlGF) promotes angiogenesis in cultured endothelial cells and stimulates retinal and systemic angiogenesis in mouse models.

- PlGF is increased in vitreous in active proliferative diabetic retinopathy (PDR) compared to control, nonangiogenic cases.

\section{What this study adds}

- PIGF is significantly downregulated in the vitreous in inactive/quiescent proliferative diabetic retinopathy, compared to active PDR.

- PIGF levels in the vitreous are not influenced by preoperative treatment with the anti-VEGF drug, bevacizumab.

- PlGF levels in the vitreous correlate closely with VEGF levels in active PDR.

- The PIGF receptor, FLT1, is expressed in vascular endothelial cells in PDR membranes.

\section{Conflict of interest}

The authors declare no conflict of interest.

\section{Acknowledgements}

This work was supported by a KKESH/JHU-WEI grant and a Research to Prevent Blindness Career Development Award (EJD). We thank Iftikhar Ahmed and Ches Souru (Department of Research, King Khaled Eye Specialist Hospital, Riyadh, KSA) for coordinating collection of specimens and patient information.

\section{References}

1 Cheung N, Mitchell P, Wong TY. Diabetic retinopathy. Lancet 2010; 376(9735): 124-136. 
2 Al-Nozha MM, Al-Maatouq MA, Al-Mazrou YY, Al-Harthi SS, Arafah MR, Khalil MZ et al. Diabetes mellitus in Saudi Arabia. Saudi Med J 2004; 25(11): 1603-1610.

3 Al-Rubeaan K, Abu El-Asrar AM, Youssef AM, Subhani SN, Ahmad NA, Al-Sharqawi AH et al. Diabetic retinopathy and its risk factors in a society with a type 2 diabetes epidemic: a Saudi National Diabetes Registry-based study. Acta Ophthalmol 2015; 93(2): e140-e147.

4 El-Asrar AM, Al-Rubeaan KA, Al-Amro SA, Kangave D, Moharram OA. Risk factors for diabetic retinopathy among Saudi diabetics. Int Ophthalmol 1998; 22(3): 155-161.

5 Yau JW, Rogers SL, Kawasaki R, Lamoureux EL, Kowalski JW, Bek T et al. Global prevalence and major risk factors of diabetic retinopathy. Diabetes Care 2012; 35(3): 556-564.

6 Aiello LP. Angiogenic pathways in diabetic retinopathy. N Engl J Med 2005; 353(8): 839-841.

7 Dewerchin M, Carmeliet P. Placental growth factor in cancer. Expert Opin Ther Targets 2014; 18(11): 1339-1354.

8 De Falco $S$. The discovery of placenta growth factor and its biological activity. Exp Mol Med 2012; 44(1): 1-9.

9 Carmeliet P, Moons L, Luttun A, Vincenti V, Compernolle V, $\mathrm{De} \mathrm{Mol} \mathrm{M}$ et al. Synergism between vascular endothelial growth factor and placental growth factor contributes to angiogenesis and plasma extravasation in pathological conditions. Nat Med 2001; 7(5): 575-583.

10 Huang H, He J, Johnson D, Wei Y, Liu Y, Wang S et al. Deletion of placental growth factor prevents diabetic retinopathy and is associated with Akt activation and HIF1alpha-VEGF pathway inhibition. Diabetes 2015; 64(1): 200-212.

11 Osaadon P, Fagan XJ, Lifshitz T, Levy J. A review of antiVEGF agents for proliferative diabetic retinopathy. Eye 2014; 28(5): 510-520.

12 Stewart MW. Pharmacokinetics, pharmacodynamics and pre-clinical characteristics of ophthalmic drugs that bind VEGF. Expert Rev Clin Pharmacol 2014; 7(2): 167-180.

13 Arimura N, Otsuka H, Yamakiri K, Sonoda Y, Nakao S, Noda $\mathrm{Y}$ et al. Vitreous mediators after intravitreal bevacizumab or triamcinolone acetonide in eyes with proliferative diabetic retinopathy. Ophthalmology 2009; 116 (5): 921-926.

14 Suzuki Y, Suzuki K, Yokoi Y, Miyagawa Y, Metoki T, Nakazawa M. Effects of intravitreal injection of bevacizumab on inflammatory cytokines in the vitreous with proliferative diabetic retinopathy. Retina 2014; 34(1): 165-171.
15 Zhao B, Cai J, Boulton M. Expression of placenta growth factor is regulated by both VEGF and hyperglycaemia via VEGFR-2. Microvasc Res 2004; 68(3): 239-246.

16 Yao YG, Yang HS, Cao Z, Danielsson J, Duh EJ. Upregulation of placental growth factor by vascular endothelial growth factor via a post-transcriptional mechanism. FEBS Lett 2005; 579(5): 1227-1234.

17 Khaliq A, Foreman D, Ahmed A, Weich H, Gregor Z, McLeod D et al. Increased expression of placenta growth factor in proliferative diabetic retinopathy. Lab Invest 1998; 78(1): 109-116.

18 Mitamura Y, Tashimo A, Nakamura Y, Tagawa H, Ohtsuka K, Mizue $Y$ et al. Vitreous levels of placenta growth factor and vascular endothelial growth factor in patients with proliferative diabetic retinopathy. Diabetes Care 2002; 25(12): 2352.

19 Grunwald JE, Daniel E, Huang J, Ying GS, Maguire MG, Toth CA et al. Risk of geographic atrophy in the comparison of age-related macular degeneration treatments trials. Ophthalmology 2014; 121(1): 150-161.

20 Bakri SJ, Moshfeghi DM, Francom S, Rundle AC, Reshef DS, Lee PP et al. Intraocular pressure in eyes receiving monthly ranibizumab in 2 pivotal age-related macular degeneration clinical trials. Ophthalmology 2014; 121(5): 1102-1108.

21 Kovacs K, Marra KV, Yu G, Wagley S, Ma J, Teague GC et al. Angiogenic and inflammatory vitreous biomarkers associated with increasing levels of retinal ischemia. Invest Ophthalmol Vis Sci 2015; 56(11): 6523-6530.

22 Campochiaro PA. Ocular neovascularization. J Mol Med 2013; 91(3): 311-321.

23 Loges S, Schmidt T, Carmeliet P. 'Antimyeloangiogenic' therapy for cancer by inhibiting PIGF. Clin Cancer Res 2009; 15(11): 3648-3653.

24 Diabetic Retinopathy Clinical Research N, Wells JA, Glassman AR, Ayala AR, Jampol LM, Aiello LP et al. Aflibercept, bevacizumab, or ranibizumab for diabetic macular edema. N Engl J Med 2015; 372(13): 1193-1203.

25 Stewart MW, Grippon S, Kirkpatrick P. Aflibercept. Nat Rev Drug Discov 2012; 11(4): 269-270.

26 Moradi A, Sepah YJ, Sadiq MA, Nasir H, Kherani S, Sophie R et al. Vascular endothelial growth factor trap-eye (Aflibercept) for the management of diabetic macular edema. World J Diabetes 2013; 4(6): 303-309.

27 Autiero M, Waltenberger J, Communi D, Kranz A, Moons L, Lambrechts D et al. Role of PIGF in the intra- and intermolecular cross talk between the VEGF receptors Flt1 and Flk1. Nat Med 2003; 9(7): 936-943. 